\title{
The Effect of Ultraviolet B on Fibrillin-1 and Fibrillin-2 in Human Non-pigmented Ciliary Epithelial Cells In Vitro
}

\author{
Yuki Shiroto ${ }^{1}$, Shingo Terashima ${ }^{1}$, Yoichiro Hosokawa ${ }^{1}$, Kyoko Oka ${ }^{2}$, \\ Keitaro Isokawa ${ }^{3}$ and Eichi Tsuruga ${ }^{1}$ \\ ${ }^{1}$ Department of Radiation Science, Graduate School of Health Sciences, Hirosaki University, 66-1 Honcho, Hirosaki, Aomori \\ 036-8564, Japan, ${ }^{2}$ Section of Pediatric Dentistry, Department of Oral Growth \& Development, Division of Clinical Dentistry, \\ Fukuoka Dental College, 2-15-1 Tamura, Sawara-ku, Fukuoka 814-0193, Japan and ${ }^{3}$ Department of Anatomy, Nihon \\ University School of Dentistry, 1-8-13, Kanda-Surugadai, Chiyoda-ku, Tokyo 101-8310, Japan
}

Received December 5, 2016; accepted May 9, 2017; published online June 14, 2017

\begin{abstract}
The ciliary zonules link the lens to the ciliary body in the eye, controlling the thickness of the lens for focusing through their characteristic elasticity. The ciliary zonules are composed of oxytalan fibers. Physiological or pathological damage to the ciliary zonules, including exposure to ultraviolet (UV)-A and UV-B components, can lead to lens dislocation. However, no studies have shown whether UV affects the ciliary zonule. Here, we assessed the effects of UV light on human nonpigmented ciliary epithelial cells (HNPCECs). HNPCECs were cultured for 4 weeks, and expression of fibrillin-1 and fibrillin-2 was confirmed. In control cultures $\left(0 \mathrm{~mJ} / \mathrm{cm}^{2}\right)$, some fibrillin-1-positive fibers were merged with fibrillin-2. After UV-A irradiation, the appearance of both fibrillin-1- and fibrillin-2-positive fibers was unchanged. However, after UV-B irradiation, fibrillin-1-positive fibers became thin at an irradiation level of $100 \mathrm{~mJ} / \mathrm{cm}^{2}$, and the fiber structure became amorphous at $150 \mathrm{~mJ} / \mathrm{cm}^{2}$. Fibrillin-2-positive fibers lost their continuity and disappeared after being exposed to $150 \mathrm{~mJ} / \mathrm{cm}^{2}$ UV-B. UV-B irradiation did not affect cell viability, possibly because of the sensitivity of fibrillin-1 and fibrillin-2 to UV-B. Thus, dislocation of the lens with age may be attributable to cumulative exposure to UV-B.
\end{abstract}

Key words: ciliary zonule, fibrillin, microfibril, ultraviolet

\section{Introduction}

Elastic system fibers include three types, i.e., elastic, elaunin, and oxytalan fibers, which differ microstructurally in their relative proportions of microfibrils and elastin [15]. Oxytalan fibers are bundles of pure microfibrils, consisting mainly of fibrillin-1 and fibrillin-2 molecules [12], which interact to form homotypic and heterotypic dimers [3, 14]. Fibrillins are assembled on or near the cell surface into microfibril arrays and then undergo maturation into transglutaminase-crosslinked microfibrils [13]. Pure oxy-

Correspondence to: Eichi Tsuruga, Department of Radiation Science, Graduate School of Health Sciences, Hirosaki University, 66-1 Honcho, Hirosaki, Aomori 036-8564, Japan. E-mail: tsuru@hirosaki.ac.jp talan fibers without elastin are distributed mainly in the ciliary zonule and in periodontal ligaments $[6,18]$.

In the eye, the ciliary zonule links the lens to the ciliary body, controlling the thickness of the lens and allowing it to focus on a target object by making use of its elasticity. In situ hybridization and immunohistochemistry in vivo have shown that nonpigmented ciliary epithelial cells on the surface of the ciliary body express fibrillin-1 [8]. Human nonpigmented ciliary epithelial cells (HNPCECs) express both fibrillin-1 and fibrillin-2 and form oxytalan fibers in vitro [20]. Structurally, the ciliary zonule and lens may be exposed cumulatively to the ultraviolet (UV) component of sunlight [16]. UV-B light (290-320 nm wavelength) is partly absorbed by ozone, whereas UV-A light (320-400 nm wavelength) is absorbed very weakly and 
transmitted easily to the Earth's surface [4]. Accordingly, our eyes can be exposed to both UV-A and UV-B.

Marfan syndrome is caused by a defect in the fibrillin-1 gene [5]. One characteristic of Marfan syndrome is lens dislocation due to disruption of the ciliary zonule [10]. Although the ciliary zonule is exposed to UV-A and UV-B in sunlight, the effects of UV on the ciliary zonule, i.e., the mechanism of degradation, have not been investigated. Therefore, to obtain basic data on the effects of UV on the ciliary zonule, we cultured HNPCECs for 4 weeks to form fibrillin-1- and fibrillin-2-positive microfibril fibers and then investigated how the fibers changed upon exposure to UV-A and UV-B irradiation.

\section{Materials and Methods}

\section{Cells and culture}

HNPCECs were purchased from Science Cell Research Laboratories (Carlsbad, CA, USA) and cultured in Dulbecco's modified Eagle medium (DMEM; SigmaAldrich, St. Louis, MO, USA) supplemented with 10\% newborn calf serum (NCS; Invitrogen), $100 \mathrm{U} / \mathrm{mL}$ penicillin, and $100 \mu \mathrm{g} / \mathrm{mL}$ streptomycin (Roche Diagnostics, Mannheim, Germany) at $37^{\circ} \mathrm{C}$ in a humidified atmosphere containing $5 \% \mathrm{CO}_{2}$. After the cells reached confluence, cells were harvested with $0.025 \%$ trypsin (Invitrogen) in PBS and transferred to plastic culture dishes at a 1:4 split ratio. For experiments, the cells were trypsinized and seeded at $1 \times 10^{6}$ cells $/ \mathrm{mL}$ in $35-\mathrm{mm}$ culture dishes (Corning Inc., Corning, NY, USA). HNPCECs reached confluence after $72 \mathrm{hr}$ (set as day 0). The HNPCECs from three different donors were used after the third to sixth passages in this study. Results among the three donors were indistinguishable.

\section{UV irradiation}

During the culture process after day 0 , the medium was refreshed every 3 days. At 4 weeks of culture, the culture medium was removed, and the cells were washed twice with phosphate-buffered saline (PBS). The medium was then replaced with $\mathrm{PBS}$ to avoid any photosensitization effect of the medium components, and UV irradiation was performed. The cells were irradiated with UV-A and UV-B at levels of $0,50,100$, and $150 \mathrm{~mJ} / \mathrm{cm}^{2}$ using a DNA-FIX (DF-312; ATTO, France), as described previously [17]. The duration of UV-A and UV-B irradiation to obtain a dose of $50 \mathrm{~mJ} / \mathrm{cm}^{2}$ was approximately $15 \mathrm{~s}$. The distance from the UV lamp to the cell culture dish was $145 \mathrm{~mm}$. After irradiation, the cells were cultured for another $24 \mathrm{hr}$ under the same culture conditions. The cells were then subjected to immunofluorescence analysis.

\section{Immunofluorescence}

HNPCECs were fixed in ice-cold $4 \%$ paraformaldehyde in PBS for 15 min and then washed with PBS. Nonspecific immunoreactivity was blocked with $1 \%$ goat serum
(Sigma, St. Louis, MO, USA) in PBS for $1 \mathrm{hr}$ at room temperature. The cell/matrix layers were then incubated for 2 $\mathrm{hr}$ at room temperature with the appropriate primary antibodies (clone 11C1.3, monoclonal antibody against human fibrillin-1 diluted 1:1000 [Thermo Fisher Scientific Anatomical Pathology, Fremont, CA, USA]; rabbit antibody against human fibrillin-2 diluted 1:1000 [Elastin Products Co., Owensville, MO, USA]). Controls were incubated with pre-immune normal mouse or rabbit $\operatorname{IgG}$ instead of the primary antibody. After being rinsed in PBS, the cells were incubated with Alexa Fluor 488-labeled goat anti-mouse IgG antibody or Alexa Fluor 568-labeled goat anti-rabbit IgG antibody (Molecular Probes, Eugene, OR, USA), diluted 1:2000 with blocking buffer, for $1 \mathrm{hr}$ at room temperature. After a final wash, the cells were stained with DAPI and viewed using an immunofluorescence microscope (IX71N-22PH; Olympus, Tokyo, Japan).

Planimetry analysis of the positive staining signals was performed using the Image $\mathbf{J}$ program (National Institutes of Health, Bethesda, MD, USA), as described previously [11]. The area of positive staining divided by the total area was calculated. Each value presented is expressed as the mean \pm standard deviation (SD).

\section{Trypan blue dye assay}

Cells were trypsinized with $0.025 \%$ trypsin (1 $\mathrm{mL} /$ well) for $5 \mathrm{~min}$ in the $\mathrm{CO}_{2}$ incubator and then neutralized with $10 \%$ NCS. The collected cell suspension was mixed with trypan blue stain solution $(1 \mathrm{~mL})$. Unstained cells (viable) were enumerated using a hematocytometer.

\section{Data analysis}

The sample number in all experiments was 15. Quantitative analysis using Image $\mathrm{J}$ was performed with TukeyKramer tests. Cell viably was analyzed by Student's t-test. All quantitative data are shown as means \pm standard deviations (SDs). Differences with $p$ values of less than 0.05 were considered significant.

\section{Results}

Under control culture conditions at a UV-A and UV-B irradiation level of $0 \mathrm{~mJ} / \mathrm{cm}^{2}$, continuous fibrillin-1-positive fibers were observed throughout the culture dish. Some of the fibrillin-2-positive fibers were merged with fibrillin-1. At all levels of UV-A irradiation $\left(50,100\right.$, and $\left.150 \mathrm{~mJ} / \mathrm{cm}^{2}\right)$, there were no differences from the control (Fig. 1). In contrast, under UV-B irradiation, the fibrillin-1-positive fibers became thin, and their continuity was disrupted at an irradiation level of $100 \mathrm{~mJ} / \mathrm{cm}^{2}$. At levels of more than 150 $\mathrm{mJ} / \mathrm{cm}^{2}$, the fiber structure became amorphous. In contrast, at $150 \mathrm{~mJ} / \mathrm{cm}^{2}$, fibrillin-2-positive fibers showed a decrease in positivity and disappeared (Fig. 2). Nuclear staining with DAPI demonstrated no changes in the shape of nuclei or in seeming the number of cells after UV-B irradiation. At the same time, area analysis using Image $\mathrm{J}$ showed that 50 


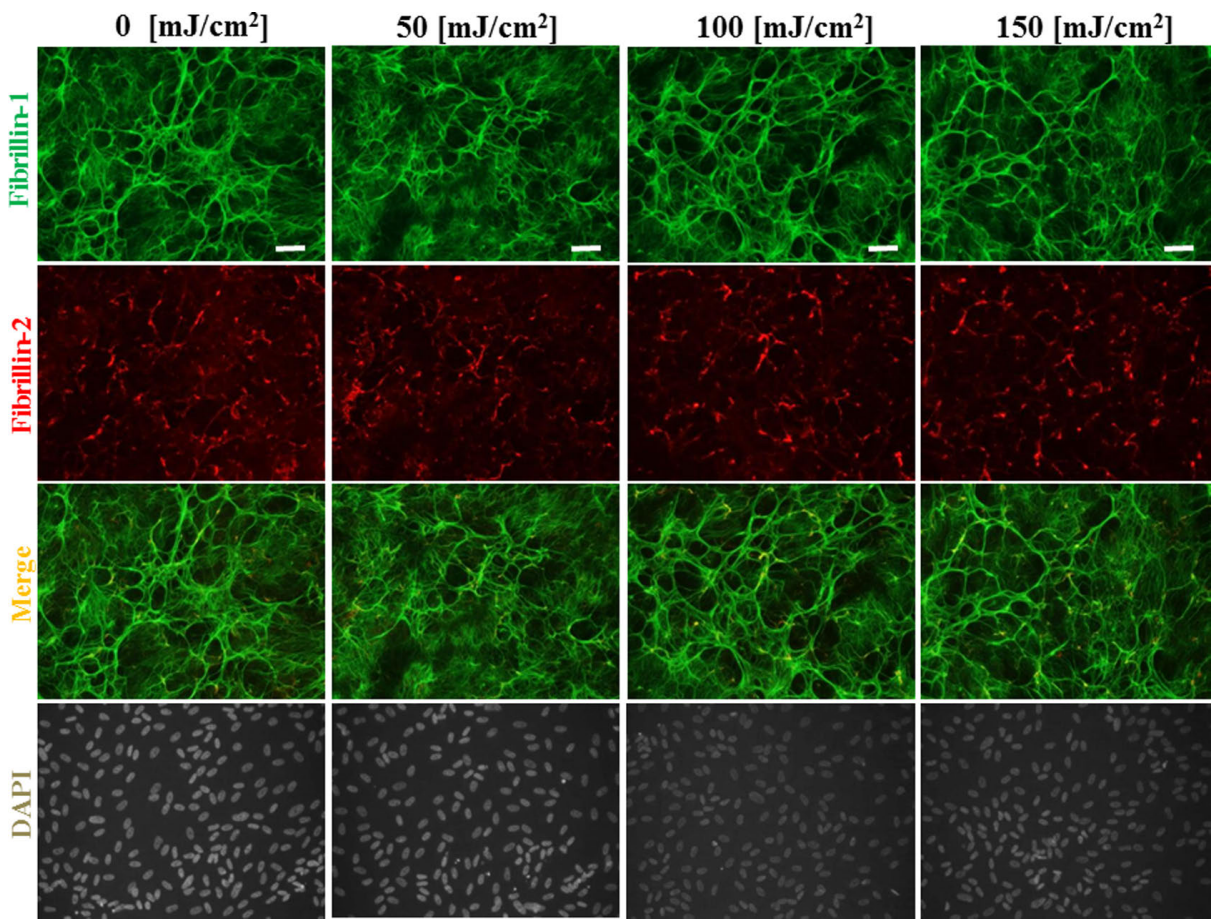

Fig. 1. Appearance of fibrillin-1 and fibrillin-2 upon exposure to UV-A irradiation. Double immunofluorescence for fibrillin-1 (upper first panels) and fibrillin-2 (second panels) in cultures of human nonpigmented ciliary epithelial cells. The cells were irradiated with the indicated dose of UV-A irradiation and were then cultured for $24 \mathrm{hr}$. Subsequently, the cells were simultaneously labeled for fibrillin-1 (green; first panels) and fibrillin-2 (red; second panels). Superimposition of both labels is shown in the third panels. DAPI was used for nuclear staining (black and white; fourth panels). Bars $=50 \mu \mathrm{m}$.

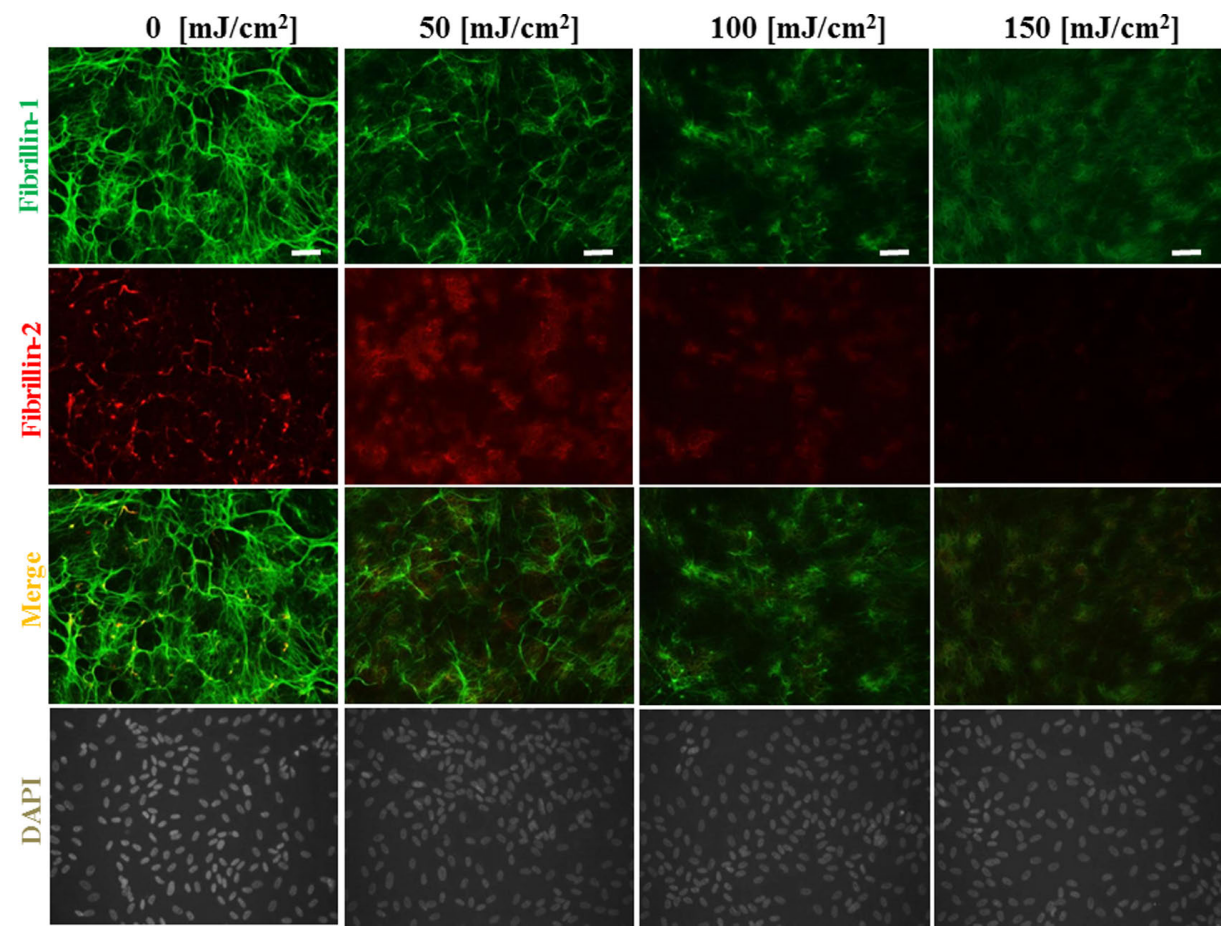

Fig. 2. Degradation of fibrillin-1 and fibrillin-2 by UV-B irradiation. Double immunofluorescence for fibrillin-1 (upper first panels) and fibrillin-2 (second panels) in cultures of human nonpigmented ciliary epithelial cells. The cells were irradiated with the indicated dose of UV-B irradiation and then cultured for $24 \mathrm{hr}$. Subsequently, the cells were simultaneously labeled for fibrillin-1 (green; first panels) and fibrillin-2 (red; second panels). Superimposition of both labels is shown in the third panels. DAPI was used for nuclear staining (black and white; fourth panels). Bars $=50 \mu \mathrm{m}$. 


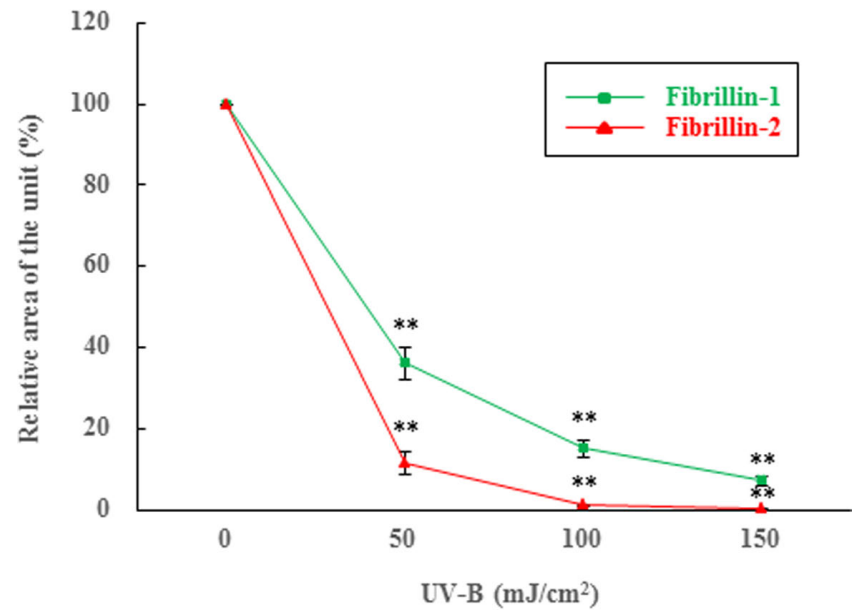

Fig. 3. Quantitative analysis of fibrillin- 1 and fibrillin-2 following UV-B irradiation. The relative areas of fibrillin-1- and fibrillin-2-positive signals shown in Fig. 2 were determined using Image J, and that at 0 $\mathrm{mJ} / \mathrm{cm}^{2}$ UV-B irradiation was set as 100 (control). Statistical analysis was performed using Tukey-Kramer tests. Data are represented as means \pm SDs $(\mathrm{n}=15) . * * p<0.01$.

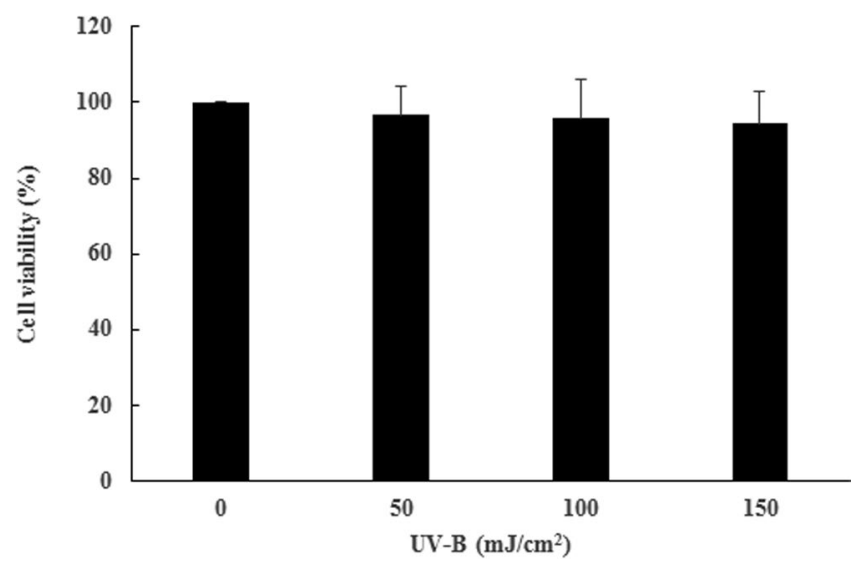

Fig. 4. Cell viability following UV-B irradiation. Viable cells were enumerated by trypan blue dye exclusion assays. Unstained cells were counted as viable. Data are expressed as means \pm SDs $(n=15)$. Significant differences were determined using unpaired Student's t-tests. Note that control does not differ significantly from any other groups.

$\mathrm{mJ} / \mathrm{cm}^{2}$ UV-B irradiation significantly reduced the level of fibrillin-1 to $36.1 \%$ of the control $\left(0 \mathrm{~mJ} / \mathrm{cm}^{2}\right)$ and that 150 $\mathrm{mJ} / \mathrm{cm}^{2} \mathrm{UV}-\mathrm{B}$ irradiation reduced the level of fibrillin-1 to $7.2 \%$ of the control. Additionally, $50 \mathrm{~mJ} / \mathrm{cm}^{2} \mathrm{UV}-\mathrm{B}$ irradiation significantly reduced the level of fibrillin-2 to $11.7 \%$ of the control $\left(0 \mathrm{~mJ} / \mathrm{cm}^{2}\right)$, and then $100 \mathrm{~mJ} / \mathrm{cm}^{2} \mathrm{UV}-\mathrm{B}$ irradiation reduced the level of fibrillin- 2 to $1.2 \%$ of the control (Fig. 3).

Next, we examined quantitatively the effects of UV-B on cell number. The number of viable cells was reduced to $94.4 \%$ of the control by $150 \mathrm{~mJ} / \mathrm{cm}^{2} \mathrm{UV}-\mathrm{B}$ irradiation. No significant differences in the number of viable cells were observed between the control $\left(0 \mathrm{~mJ} / \mathrm{cm}^{2}\right)$ and each UV-B irradiation dose (50, 100, and $150 \mathrm{~mJ} / \mathrm{cm}^{2}$; Fig. 4).

\section{Discussion}

Here, we demonstrated, for the first time, that oxytalan fibers were degraded upon exposure to UV-B at 150 $\mathrm{mJ} / \mathrm{cm}^{2}$, but were unaffected by exposure to UV-A because the decrease and disappearance of the immunofluorescent signal was observed only in UV-B-irradiated cells.

UV-B has been reported to induce secretion of matrix metalloproteinase (MMP)-2 by human dermal fibroblasts [2]. Additionally, UV-B irradiation of human dermal fibroblasts has been shown to induce MMP-2 secretion and suppress elastin deposition at irradiation levels of 40-100 $\mathrm{mJ} / \mathrm{cm}^{2}$ [7]. Thus, activated MMP-2 is thought to degrade elastin. The sites of fibrillin-1 degradation by MMP-2 have been identified [1]. Moreover, we have previously shown that HNPCECs express MMP-2 and that MMP-2 activated by concanavalin A degrades fibrillin-1 and fibrillin-2 in cell matrix layers [11]. The degradation patterns of fibrillin-1 and fibrillin-2 observed in this study were similar temporally to those in our previous study [11], in which we found that fibrillin-2 was preferentially degraded. Moreover, UV$B$ irradiation of an epidermal cell line at $180 \mathrm{~mJ} / \mathrm{cm}^{2}$ was shown to induce the expression of inflammatory cytokines, such as tumor necrosis factor- $\alpha$ and interleukin-1 $\beta$ [21]. Therefore, the degradation of oxytalan fibers observed upon exposure to UV-B irradiation may be caused by MMP-2 activation. We are currently investigating whether UV-B activates MMP-2 in cultured HNPCECs.

The ciliary zonule is composed of fibrillin-based microfibrils $[9,18]$. Rotary shadowing images of fibrillinbased microfibrils extracted from COS-1 cell/matrix layers exhibit beaded filaments with a periodicity of $56 \mathrm{~nm}$ [19]. These observations suggest that UV-B irradiation increases the periodicity of microfibrils and induces fragmentation in a dose-dependent manner over a range of 0 to $100 \mathrm{~mJ} / \mathrm{cm}^{2}$. The triple helical $[\alpha 1(\mathrm{I})]_{2} \alpha 2(\mathrm{I})$ band of type I collagen was not affected by UV-B irradiation between 1 and 500 $\mathrm{mJ} / \mathrm{cm}^{2}$. Moreover, human dermal fibroblasts irradiated with UV-A at a level of $10 \mathrm{~mJ} / \mathrm{cm}^{2}$ followed by $50 \mathrm{~mJ} / \mathrm{cm}^{2}$ UV-B at 24-hr intervals led to a reduction of fibrillin-1 and microfibril-associated glycoprotein (MAGP)-1 [22], mimicking the conditions occurring in vivo. Therefore, the sensitivity of oxytalan fibers and collagen fibers to UV-B appears to differ, suggesting that the observed changes in microfibril microstructure may be explained by loss of oxytalan fiber continuity, as demonstrated in our immunofluorescence study with irradiation at $150 \mathrm{~mJ} / \mathrm{cm}^{2}$. The sensitivity of the various molecules associated with oxytalan fibers, such as MAGP-1 and EMILIN-1, should be considered when assessing the mechanisms responsible for disruption of the ciliary zonule by UV irradiation.

In summary, fibrillin-1-positive fibers were shown to lose their continuity and become thin upon exposure to UV-B at an irradiation level of $100 \mathrm{~mJ} / \mathrm{cm}^{2}$, and the fiber structure became amorphous at $150 \mathrm{~mJ} / \mathrm{cm}^{2}$. Notably, the observation that fibrillin-2-positive fibers lost their continu- 
ity and disappeared at an irradiation level of $150 \mathrm{~mJ} / \mathrm{cm}^{2}$ may reflect the respective sensitivities of fibrillin-1 and fibrillin-2 in the ciliary zonule to UV-B. This finding suggested that dislocation of the lens in later life may be explained by cumulative exposure to UV-B.

\section{Acknowledgments}

We thank Dr. Hironori Yoshino (Department of Radiation Science, Graduate School of Health Sciences, Hirosaki University) for helpful discussions. This work was supported by JSPS KAKENHI Grant Number JP15K11001.

\section{References}

1. Ashworth, J. L., Murphy, G., Rock, M. J., Sherratt, M. J., Shapiro, S. D., Shuttleworth, C. A. and Kielty, C. M. (1999) Fibrillin degradation by matrix metalloproteinases: implications for connective tissue remodelling. Biochem. J. 340; 171-181.

2. Brenneisen, P., Sies, H. and Scharffetter-Kochanek, K. (2002) Ultraviolet-B irradiation and matrix metalloproteinases: from induction via signaling to initial events. Ann. N. Y. Acad. Sci. 973; 31-43.

3. Charbonneau, N. L., Dzamba, B. J., Ono, R. N., Keene, D. R., Corson, G. M., Reinhardt, D. P. and Sakai, L. Y. (2003) Fibrillins can co-assemble in fibrils, but fibrillin fibril composition displays cell-specific differences. J. Biol. Chem. 278; 2740 2749.

4. Crutzen, P. J. (1992) Ultraviolet on the increase. Nature 356; 104-105.

5. Dietz, H. C., Ramirez, F. and Sakai, L. Y. (1994) Marfan's syndrome and other microfibrillar diseases. Adv. Hum. Genet. 22; 153-186.

6. Fullmer, H. M. and Lillie, R. D. (1958) The oxytalan fiber: a previously undescribed connective tissue fiber. J. Histochem. Cytochem. 6; 425-430.

7. Ham, S. A., Yoo, T., Hwang, J. S., Kang, E. S., Paek, K. S., Park, C., Kim, J. H., Do, J. T. and Seo, H. G. (2014) Peroxisome proliferator-activated receptor delta modulates MMP-2 secretion and elastin expression in human dermal fibroblasts exposed to ultraviolet B radiation. J. Dermatol. Sci. 76; 44-50.

8. Hanssen, E., Franc, S. and Garrone, R. (2001) Synthesis and structural organization of zonular fibers during development and aging. Matrix Biol. 20; 77-85.

9. Hiraoka, M., Inoue, K., Ohtaka-Maruyama, C., Ohsako, S., Kojima, N., Senoo, H. and Takada, M. (2010) Intracapsular organization of ciliary zonules in monkey eyes. Anat. Rec. (Hoboken) 293; 1797-1804.

10. Judge, D. P. and Dietz, H. C. (2005) Marfan's syndrome. Lancet
366; 1965-1976.

11. Kawagoe, M., Tsuruga, E., Oka, K., Sawa, Y. and Ishikawa, H. (2013) Matrix metalloproteinase-2 degrades fibrillin-1 and fibrillin-2 of oxytalan fibers in the human eye and periodontal ligaments in vitro. Acta Histochem. Cytochem. 46; 153-159.

12. Kielty, C. M. (2006) Elastic fibres in health and disease. Expert Rev. Mol. Med. 8; 1-23.

13. Kielty, C. M., Baldock, C., Lee, D., Rock, M. J., Ashworth, J. L. and Shuttleworth, C. A. (2002) Fibrillin: from microfibril assembly to biomechanical function. Philos. Trans. R. Soc. Lond. B. Biol. Sci. 357; 207-217.

14. Lin, G., Tiedemann, K., Vollbrandt, T., Peters, H., Batge, B., Brinckmann, J. and Reinhardt, D. P. (2002) Homo- and heterotypic fibrillin-1 and -2 interactions constitute the basis for the assembly of microfibrils. J. Biol. Chem. 277; 50795-50804.

15. Mecham, R. P. and Davis, E. C. (1994) Elastic fiber structure and assembly. In "Extracellular Matrix Assembly and Structure" ed. by P. D. Yurchenco, D. E. Birk and R. P. Mecham, Academic Press, New York, pp. 281-314.

16. Narayanan, P., Merriam, J. C., Vazquez, M. E. and Dillon, J. (1996) Experimental model of light focusing of the peripheral cornea. Invest. Ophthalmol. Vis. Sci. 37; 37-41.

17. Niimura, Y., Moue, T., Takahashi, N. and Nagai, K. (2008) Screening of biomarker genes activated by irradiation of ultraviolet B rays in mouse lymph node M10 cells. J. Radiat. Res. 49; 635-644.

18. Raviola, G. (1971) The fine structure of the ciliary zonule and ciliary epithelium. With special regard to the organization and insertion of the zonular fibrils. Invest. Ophthalmol. 10; 851-869.

19. Sherratt, M. J., Bayley, C. P., Reilly, S. M., Gibbs, N. K., Griffiths, C. E. and Watson, R. E. (2010) Low-dose ultraviolet radiation selectively degrades chromophore-rich extracellular matrix components. J. Pathol. 222; 32-40.

20. Yamanouchi, K., Tsuruga, E., Oka, K., Sawa, Y. and Ishikawa, H. (2012) Fibrillin-1 and fibrillin-2 are essential for formation of thick oxytalan fibers in human nonpigmented ciliary epithelial cells in vitro. Connect. Tissue Res. 53; 14-20.

21. Yin, Z., Xu, J., Zhou, B., Wu, D., Xu, Y., Zhang, J. and Luo, D. (2014) Topical pimecrolimus inhibits high-dose UVB irradiationinduced epidermal Langerhans cell migration, via regulation of TNF-alpha and E-cadherin. Drug Des. Devel. Ther. 8; 18171825.

22. Zheng, Q., Chen, S., Chen, Y., Lyga, J., Wyborski, R. and Santhanam, U. (2013) Investigation of age-related decline of microfibril-associated glycoprotein-1 in human skin through immunohistochemistry study. Clin. Cosmet. Investig. Dermatol. 6; 317-323.

This is an open access article distributed under the Creative Commons Attribution License, which permits unrestricted use, distribution, and reproduction in any medium, provided the original work is properly cited. 